\title{
DOUBLE LOOPS AND TERNARY RINGS
}

\author{
BY PETER WILKER \\ Communicated by E. Hewitt, February 10, 1964
}

1. Let $T$ be a ternary ring with the ternary operation $T(a, b, c)$ and the distinguished elements 0,1 (see [4]). On $T$, two loop structures can be defined by means of the binary operations $a+b=T(a, 1, b)$ and $a b=T(a, b, 0)$. The resulting loops are called the additive and the multiplicative loop of $T$, respectively. Together with $a 0=0 a=0$ they define the structure of a double loop on $T$, which satisfies moreover the following condition:

(1) For every $a, b \in T, b \neq 1$, the equation $x+a=x b$ is uniquely solvable.

The question arises whether any double loop, satisfying the necessary condition (1), can be the double loop of a ternary ring. Hughes [3] has answered this question for countably infinite loops, but with another definition of addition on $T$.

The purpose of this note is to present the affirmative answer to the question in the infinite case: any infinite double loop satisfying (1) can be given canonically the structure of a ternary ring (Theorem 1 below). It will also be established (Theorems $2 \mathrm{~m}$ and $2 \mathrm{a}$ ) that any infinite loop can be the additive or the multiplicative loop of a double loop satisfying (1), and hence of a ternary ring (see [2, I 4]).

Important special cases of ternary rings are those in which the ternary operation can be expressed as a linear combination of the two binary ones: $T(a, b, c)=a b+c$. In this note, such rings will be called linear. The double loop of a linear ternary ring satisfies the following conditions:

(2) For every $b, b^{\prime}, c, c^{\prime} \in T, b \neq b^{\prime}$, the equation $x b+c=x b^{\prime}+c^{\prime}$ is uniquely solvable.

(3) For every $a, a^{\prime}, d, d^{\prime} \in T, a \neq a^{\prime}$, the equations $a x+y=d$, $a^{\prime} x+y=d^{\prime}$ are solvable.

Conversely, any double loop satisfying (2), (3) can be made into a linear ternary ring by defining $T(a, b, c)=a b+c$.

The question, what loops can be additive loops of double loops satisfying (2), (3) has been answered by Hughes [3] for the case of countably infinite groups.

In this note, sufficient conditions will be given for an infinite loop (of any cardinality) to be the additive loop of a double loop satisfying (2), (3), and therefore of a linear ternary ring (Theorem 3). The conditions cover the case of infinite groups. 
On the other hand, the construction of infinite loops which cannot be the additive loops of linear ternary rings will be described (Theorem 4 ), thus confirming - and extending to arbitrary infinite cardinality-a conjecture by Hughes [3].

Full details of the proofs will appear elsewhere.

2. Let $S$ be a set, containing a distinguished element $d$. Suppose to each $s \in S$ there corresponds a bijective mapping $M(s)$ of $S$ onto itself, $M(d)$ being the identity mapping; suppose furthermore that given $f, g \in S$, there is exactly one $s \in S$ such that $M(s) f=g$. Then the collection $M(s), s \in S$, will be called a simply transitive $(S, d)$-family.

Now let $L$ be an infinite double loop with neutral elements 0,1 , satisfying (1). Assume $L$ well-ordered according to the initial number $\delta$ corresponding to the cardinal of $L$ (see [1]). The elements of $L$ can then be indexed as $l_{\lambda}, 0 \leqq \lambda<\delta$, with $l_{0}=0, l_{1}=1$.

Theorem 1. To every ordinal $\gamma, 1 \leqq \gamma<\delta$, there exists a simply transitive $(L, 0)$-family $M_{\gamma}(u), u \in L$, satisfying

(a) $M_{\gamma}(u) 0=u l_{\gamma}$ for all $u \in L$.

(b) For every pair of ordinals $\alpha, \beta, 1 \leqq \alpha<\beta<\delta$, the mappings $M_{\alpha}(u)^{-1} M_{\beta}(u), u \in L$, form a simply transitive $(L, 0)$-family.

(c) For every ordinal $\gamma, 1 \leqq \gamma<\delta$, and for every pair of elements $f, g \in L, f \neq 0, g \neq l_{\gamma}$, there is exactly one $u \in L, u \neq 0$, such that $M_{0}(u)^{-1} M_{\gamma}(u) f=g$, where $M_{0}(u)$ is a mapping defined for all $u \neq 0$ by $M_{0}(u) f=u f$.

(d) Given elements $u_{1}, u_{2}, v_{1}, v_{2} \in L, u_{1} \neq u_{2}, v_{1} \neq v_{2}$, there is an ordinal $\gamma, 1 \leqq \gamma<\delta$, and an $f \in L$ such that $M_{\gamma}\left(u_{1}\right) f=v_{1}, M_{\gamma}\left(u_{2}\right) f=v_{2}$.

(e) $M_{1}(u) f=u+f$ for all $u, f \in L$.

Defining the ternary operation $T(a, b, c)$ on $L$ by $T(a, b, c)=M_{\gamma}(a) c$, if $b=l_{\gamma} \neq 0$, and by $T(a, b, c)=c$, if $b=0$, it is easily seen that $T$ satisfies the axioms imposed on such an operation in a ternary ring. Because of (a) and (e) the double loop of this ternary ring is isomorphic to the given one.

3. If $L_{m}$ consists of a (multiplicatively written) infinite loop $L$ with neutral element 1 and of an additional element 0 , for which $a 0=0 a=0$ has been defined, a slight rearrangement of part of the proof of Theorem 1 yields

TheOREM $2 \mathrm{~m}$. There exists a simply transitive $\left(L_{m}, 0\right)$-family $M(u)$, $u \in L_{m}$, satisfying

(a) $M(u) 0=u$ for all $u \in L_{m}$.

(b) Given $f, g \in L_{m}, f \neq 0, g \neq 1$, there is exactly one $u \neq 0$ such that 
$M_{0}(u)^{-1} M(u) f=g$, where $M_{0}(u)$ is a mapping defined for all $u \neq 0$ by $M_{0}(u) f=u f$.

Clearly, $M(a) b=a+b$ defines an addition on $L_{m}$ which makes $L_{m}$ into a double loop with neutral elements 0 and 1, satisfying (1).

Writing the same infinite loop $L$ additively, with neutral element 0 , denote by $L_{a}$ the set of elements of $L$ other than 0 , and choose an element of $L_{a}$ as 1 .

Theorem 2a. There exists a simply transitive $\left(L_{a}, 1\right)$-family $M(u)$, $u \in L_{a}$, satisfying

(a) $M(u) 1=u$ for all $u \in L_{a}$.

(b) Given $f, g \in L_{a}, f \neq 1$, there is exactly one $u \neq 0$ such that $M_{0}(u)^{-1} M(u) f=g$, where $M_{0}(u)$ is defined for all $u \in L$ by $M_{0}(u) f$ $=u+f$.

As above, $M(a) b=a b$ defines a multiplicative loop structure on $L_{a}$, with 1 as neutral element, and together with $a 0=0 a=0$ a double loop structure on $L$, satisfying (1).

4. In this section $L$ again denotes an additively written infinite loop. Consider the equations in $x, y$ :

$$
\begin{aligned}
& a+x=c+y, \\
& b+x=d+y
\end{aligned}
$$

where $a, b, c \in L$, and let $D(a, b, c)$ be the set of all those elements $d \in L$ for which equations (4) are solvable. $D(a, b, c)$ is of course never empty, or card $D(a, b, c) \geqq 1$ (cardinal numbers are assumed to be comparable).

Suppose $L$ has the following properties: there exist cardinal numbers $k, k^{\prime}$, both strictly less than card $L$, such that

(5) card $D(a, b, c) \leqq k$ for all $a, b, c \in L$.

(6) For every $a, b$, the set of all $c$, and for every $a, c$, the set of all $b$, for which card $D(a, b, c)>1$, have cardinalities less than or equal to $k^{\prime}$.

It is easily seen that groups are characterized by card $D(a, b, c)$ $=1$ for all $a, b, c \in L$; consequently, the family of loops satisfying (5), (6) contains all infinite groups.

With $L_{a}$ as the set of elements of $L$ other than 0 , and with $1 \in L_{a}$ chosen arbitrarily, the following theorem holds:

THEOREM 3. If $L$ is an infinite loop satisfying (5), (6), there exists a simply transitive $\left(L_{a}, 1\right)$-family $M(u), u \in L_{a}$, with

(a) $M(u) 1=u$ for all $u \in L_{a}$. 
(b) To every $r, r^{\prime} \in L, r \neq r^{\prime}$, and every $f, f^{\prime} \in L_{a}, f \neq f^{\prime}$, there is exactly one $u \in L_{a}$ with $M(u) f=g, M(u) f^{\prime}=g^{\prime}$, where $g, g^{\prime} \in L_{a}$ are such that $g+r=g^{\prime}+r^{\prime}$.

(c) To every $r, r^{\prime} \in L, r \neq r^{\prime}$, and every $u, u^{\prime} \in L_{a}, u \neq u^{\prime}$, there is an $f \in L_{a}$ with $M(u) f=g, M\left(u^{\prime}\right) f=g^{\prime}$, where $g, g^{\prime} \in L_{a}$ are such that $g+t=r$, $g^{\prime}+t=r^{\prime}$ for an appropriately chosen $t \in L$.

Defining multiplication on $L_{a}$ by $M(a) b=a b$ and supplementing it on $L$ by $a 0=0 a=0, L$ is seen to acquire the structure of a double loop satisfying (2), (3), and therefore also the structure of a linear ternary ring.

5. Theorem 4. There exist (additively written) infinite loops $L$ of any cardinality, containing three mutually distinct, nonzero elements $a, b, c$ with the property that equations (4) are solvable for any $d \in L$, $d \neq b, c$.

A loop of this kind cannot be the additive loop of a linear ternary ring. For if $a, b, c$ are three distinct, nonzero elements of such a ring, and if $u$ and $d$ are defined by $u a=b, d=u c$, then $u \neq 1$ and $d \neq b, c$. If equations (4) were solvable with these $a, b, c, d$, one could write

contradicting (2).

$$
\begin{aligned}
& 1 a+x=1 c+y, \\
& u a+x=u c+y,
\end{aligned}
$$

6. The proofs of Theorems 1 to 4 are based essentially on the following lemma. Let $\gamma, \delta$ denote ordinals, $2 \leqq \gamma<\delta, \delta$ transfinite. Suppose to every ordinal $\lambda, 0 \leqq \lambda<\gamma$ there correponds a set $B^{\lambda}$, consisting of ordinals less than $\delta$. The sequence of type $\gamma$ (see [1]): $b=\left(B^{0}, B^{1}, \cdots, B^{\lambda}, \cdots\right)$ will be called a bracket (of breadth $\gamma$ ), and the set $B^{\lambda}$ its $\lambda$-component. Let $B=B(\delta, \gamma)$ denote the set of all such brackets of given breadth and given range $\delta$.

In the sequel $S$ will denote a subset of $B, \rho, \lambda$ ordinals with $0 \leqq \rho<\delta$, $0 \leqq \lambda<\gamma$, and $S(\rho, \lambda)$ will have the meaning of the set of all brackets in $S$ containing $\rho$ in their $\lambda$-components, card $\delta$ is the cardinal number corresponding to the ordinal $\delta$. $S$ will be called a Latin chain (of length $\delta$ and breadth $\gamma$ ) provided card $S(\rho, \lambda)=1$ for every pair $\rho, \lambda$.

Assume now that a subset $S \subset B$ has the following properties:

(7) $\operatorname{card} S(\rho, \lambda) \geqq$ card $\delta$ for all $\rho, \lambda$.

(8) If $U$ is a subset of $S$ with card $U<$ card $\delta$, the union of the $\lambda$ components of the members of $U$ does not contain all ordinals less than $\delta$.

(9) Given $\rho, \lambda$ and a bracket $b \in S, b \in S(\rho, \lambda)$, let $S(\rho, \lambda, b)$ be the 
subset of all those brackets in $S(\rho, \lambda)$ having at least one ordinal in common with bracket $b$ in each of two corresponding components. Then for fixed $\rho, \lambda$, card $S(\rho, \lambda, b)$ is bounded from above for all $b \notin S(\rho, \lambda)$, and the least upper bound is strictly less than card $\delta$.

Leмma. Let $\delta$ be an initial number, $S$ a subset of $B$ satisfying (7) to (9), let $T \subset S$ with card $T<$ card $\delta$, and suppose $T$ has the property that card $T(\rho, \lambda) \leqq 1$, where $T(\rho, \lambda)$ has the same meaning as $S(\rho, \lambda)$ above. Then $T$ can be extended within $S$ to a Latin chain.

The results contained in this note were obtained during a leave of absence from the University of Bern, Switzerland. The author is happy to have this opportunity to express his gratitude to the authorities of the Canton and the University of Bern, and to Professors Hadwiger, Nef and Scherrer, for their generosity and for their encouragement.

\section{REFERENCES}

1. H. Bachmann, Transfinite Zahlen, Ergebnisse der Mathematik und ihrer Grenzgebiete, Heft 1, Springer, Berlin, 1955.

2. R. H. Bruck, $A$ survey of binary systems, Ergebnisse der Mathematik und ihrer, Grenzgebiete, Heft 20, Springer, Berlin, 1958.

3. D. R. Hughes, Additive and multiplicative loops of planar ternary rings, Proc. Amer. Math. Soc. 6 (1955), 973-980.

4. G. Pickert, Projective Ebenen, Grundlehren der mathematischen Wissenschaften in Einzeldarstellungen mit besonderer Ber ücksichtigung der Anwendungsgebiete, Bd. 80, Springer, Berlin, 1955.

University OF BERN, SwitzerLand AND State University of New York at Buffalo 